\title{
NÍVEL DE ATIVIDADE FÍSICA E MASSA CORPORAL EM ADOLESCENTES DE ESCOLAS PÚBLICAS E PRIVADAS DE TAGUATINGA-DF
}

\author{
Level of physical activity and body mass in teens of public \\ and private schools of Taguatinga-DF
}

\author{
Luciana Santos de Oliveira ${ }^{1,2,3}$ \\ ${ }^{1}$ Pós-graduação Strictu Senso da Universidade Católica de Brasília (UCB-DF). \\ ${ }^{1}$ Faculdade Albert Einstein (FALBE-DF). \\ ${ }^{3}$ Universidade Estadual de Santa Cruz (UESC-BA)
}

\begin{abstract}
Resumo: A composição corporal é um indicador antropométrico fortemente associado à manifestação de doenças crônico-degenerativas, sendo influenciado por componentes do estilo de vida, como o nível de atividade física. Fundamentado no exposto, o propósito deste estudo foi analisar o nível de atividade física (NAF) e a massa corporal de adolescentes de escolas públicas e privadas de Taguatinga-DF. A amostra foi constituída

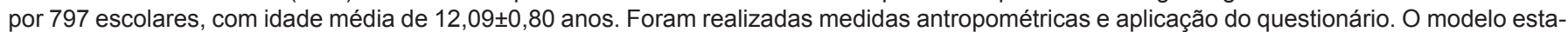
tístico utilizado foi o da estatística descritiva e o teste qui-quadrado $\left(\mathrm{x}^{2}\right)$. Os resultados revelam uma porcentagem alta de jovens com excesso de peso; sobrepeso, $12,3 \%$, e obeso, 3,1\%. O NAF mostra que os alunos da escola pública são mais ativos (19,4\%) e com a relação do IMC revela que a maioria dos alunos com excesso de peso encontra-se sedentária. A situação dos estudantes sugere ações intervencionistas que venham incentivar a prática de atividade física.
\end{abstract}

Palavras-chave: Atividade física, excesso de peso, sedentarismo.

Abstract: The body composition is a strong anthropometric indicator associated with clinical manifestations of chronic-degenerative diseases. It is influenced by components of lifestyle such as levels of physical activity. The purpose of this study was to evaluate the level of physical activity (LFA) and body mass index (BMI) of each individual in a group of teenage students in both public and private schools in Taguatinga-DF. The sample was constituted by 797 with average age of $12,09 \pm 0,80$ years old. It was accomplished anthropometric measures as well as an application of a questionnaire. The statistical model used was the descriptive statistics besides the qui-square $\left(\mathrm{x}^{2}\right)$ test. The results show a raising percentage of young people with weight excess; overweight, 12,3\%; and obesity, 3,1\%. LPA shows that the public school students are more active $19,4 \%$ and with BMI relation reveals that most students with overweight is sedentary. The students' situation suggests interventionist legal actions that come to encourage the practice of physical activity.

Keywords: Physical activity - overweight - sedentary.

Aceito em 19/02/13 - Revista de Educação Física 2013 Abr; 157:30-37. Rio de Janeiro - Brasil

\section{INTRODUÇÃO}

A atividade física regular é entendida como o movimento corporal produzido pela contração do músculo esquelético que aumenta o gasto de energia acima do nível basal ${ }^{\left({ }^{30}\right)}$. É uma área relevante para estudos científicos, pois apresenta relação inversa com as doenças crônico-degenerativas, isto é, indivíduos fisicamente ativos tendem a apresentar menor mortalidade e morbidade por essas doenças ${ }^{(9)}$. Desta forma, é importante enfatizar que apenas 30 minutos do exercício por dia podem ajudar a impedir e tratar diversas condições crônicas ${ }^{(3)}$. É de suma importância a adoção de atividade física regular, para melhoria dos níveis de saúde e, especialmente, para a prevenção de doenças ${ }^{(20)}$.
A inatividade física é um fator que influi no aparecimento da obesidade ${ }^{(15)}$. Cerca de $70 \%$ da população mundial é inativa, sendo que, só no Brasil, há um índice de $60 \%$. Como na maioria dos países, no Brasil, há uma tendência crescente de as pessoas tornarem-se cada vez mais sedentárias, esquecendo-se que a atividade física, além de prevenir o excesso de peso, é também benéfica para a saúde mental e emocional ${ }^{(1)}$. A obesidade é um problema mundial e pode ser classificada pela análise da massa corporal, conceituada como uma medida antropométrica que expressa a dimensão da massa ou volume corporal ${ }^{(26)}$. A mais favorável medida de massa corporal tradicionalmente tem sido o peso isolado ou peso ajustado para a altura (IMC). Pesquisas sobre o perfil do IMC idade e sexo, ob- 
tidos através de pesquisas, propuseram limites de sobrepeso e obesidade para a faixa etária de 2 a 20 anos para uso internacional ${ }^{(10)}$, o que significa um avanço na assistência à saúde de crianças e adolescentes ${ }^{(28)}$.

No mundo, ocorre o aumento de pessoas obesas ${ }^{(20)}$, e, nos últimos 30 anos, aproximadamente 400 milhões de pessoas no mundo tornaram-se obesas, estando o Brasil está entre esses países, com $43 \%$ de excesso de peso na população ${ }^{(22)}$, sendo que $13,0 \%$ dos adolescentes em Brasília estão na mesma situação ${ }^{(16)}$, com excesso de peso e obesidade, definidos como excessiva acumulação de gordura que pode danificar a saúde ${ }^{(31)}$.

Existem estudos mostrando níveis baixos de atividade física e prevalência de excesso de peso, uma delas, com o propósito de analisar os indicadores de risco para hipertensão arterial em crianças e adolescentes da cidade de Fortaleza (CE), que detectou $51,4 \%$ da amostra como sedentários, além de uma prevalência de $16,8 \%$ de excesso de peso ${ }^{(4)}$. Outro estudo, envolvendo adolescentes da cidade de Pelotas (Brasil), confirma essa problemática, quando descreve o nível de atividade física e avalia o efeito de variáveis socioculturais sobre o nível de atividade física. O resultado mostra uma grande tendência ao sedentarismo, com $48,7 \%$ dos meninos e $67,5 \%$ das meninas inativos ${ }^{(13)}$.

Como a atividade física realizada na infância e na adolescência tende a permanecer na vida adulta, são de fundamental importância um melhor conhecimento dos grupos populacionais de risco, a identificação dos hábitos não saudáveis e as suas causas, para que políticas e programas de saúde sejam implementados, visando a um melhor controle das doenças crônico-degenerativas da vida adulta ${ }^{(18)}$.

Diante desse grave problema, este estudo teve como propósito identificar e relacionar o nível de atividade física e a massa corporal em adolescentes de escolas públicas e privadas de Taguatinga-DF, através do projeto Estilo de Vida.

\section{MÉTODOS}

A pesquisa foi realizada em Taguatinga-DF com a amostra constituída de 797 estudantes, com idades de 11 a 13 anos, 580 adolescentes das es- colas públicas e 217 das privadas. Para atingir a população estudantil de pontos diversos da cidade, ela foi dividida geograficamente em três regiões: norte, centro e sul. Foram selecionadas seis escolas, duas de cada região, sendo uma escola pública e a outra privada, para assegurar maior representatividade à amostra. A escolha das seis escolas, três públicas e três privadas, pautou-se na receptividade e no maior nível de aceitação ao projeto.

Houve três momentos iniciais para apresentação do projeto Estilo de Vida. Em primeiro lugar, a pesquisadora entrou em contato com os diretores de cada escola para explicar os objetivos da pesquisa e a importância do estudo. Após o aceite dos diretores, com o mesmo objetivo, foi feita uma reunião, previamente marcada, com os professores de cada escola selecionada. Por fim, ocorreu um encontro com os alunos de cada escola, para apresentação do projeto, explicando os objetivos, a importância e os procedimentos. Nessa mesma ocasião, foi entregue a cada aluno um termo livre e esclarecido para ser assinado pelos pais.

A coleta de dados foi realizada em duas etapas. Na primeira, foi feita uma avaliação antropométrica, a qual nos forneceu informações sobre a classificação dos adolescentes quanto ao IMC. Os materiais utilizados foram: balança digital da marca Plenna $\AA_{\text {, }}$ (com precisão de $0,01 \mathrm{~g}$ ) para a massa corporal. $\mathrm{O}$ avaliado posicionou-se em pé, no centro da plataforma da balança, descalço, usando roupas leves, de costas para o visor da balança, permanecendo imóvel durante o exame. Em seguida, realizou-se a medida da estatura, usando-se um estadiômetro da marca $S e c a \circledR$, (com resolução de 0,01 c). O avaliado foi mensurado descalço, em posição ereta, encostado numa superfície plana vertical, braços pendentes, os calcanhares unidos e as pontas dos pés afastadas, formando um ângulo de $60^{\circ}$, joelhos em contato, cabeça ajustada ao plano de Frankfurt e em inspiração profunda.

Os adolescentes foram classificados de acordo com o IMC/idade 10, com pontos de corte para sobrepeso e obesidade em meninas e meninos de 2 a 18 anos, baseado em estudos transversais representativos de seis países, dentre eles o Brasil, cada um com mais de 10 mil participantes. Os pontos de corte foram ajustados em percentis 85 e 95. 
$\mathrm{Na}$ segunda etapa, os estudantes responderam um questionário de estilo de vida ${ }^{(6)}$, para classificar o nível de atividade física. A aplicação ocorreu na parte da manhã, em sala de aula, pela própria pesquisadora, com auxílio de dois estagiários. Os alunos foram orientados sobre o conteúdo do questionário, e os alunos respondiam cada questão calmamente e só seguiam para a questão seguinte, quando todos tivessem respondido à anterior.

Foram excluídos os escolares que faltaram à segunda etapa do estudo, ou não preencheram o questionário corretamente.

Para o cálculo do nível de atividade física, foram considerados: tipo de atividade física, intensidade do esforço físico (leve, moderado, vigoroso) e a freqüência semanal. Foram feitas a somatória e a multiplicação das atividades físicas e freqüência semanal na equação abaixo: $[($ dança*freqüência semanal) + (futebol*freqüência semanal) + (ciclismo*freqüência semanal) + (tarefas domésticas*freqüência semanal) + (lazer1*freqüência semanal) + (lazer2*freqüência semanal) + (lazer3*freqüência semanal)]. Para conversão das informações obtidas na atividade física em valores estimados de dispêndio energético, recorreu-se ao compêndio de atividades físicas que oferece informações sobre o gasto energético, em unidades do equivalente metabólico de trabaIho para uma atividade particular (MET) para quase quinhentas formas de atividades físicas ${ }^{(2)}$.

$\mathrm{Na}$ seqüência, estabeleceu-se ponto de cortes por meio do escore geral, obtido na soma das atividades físicas, usando-se a classificação de quartis, sendo consideradas inativas fisicamente as crianças com escore abaixo do percentil 25; insuficientemente ativas (percentil entre 25 e 50), moderadamente ativas (percentil entre 50 e 75) e ativas para valores acima do percentil 75 .

Trata-se de um estudo transversal, aprovado pelo Comitê de Ética em Pesquisa da Universidade Católica de Brasília sob o número 34 CEP/UCB 2007 e acompanha normas da Resolução 196/96 do Conselho Nacional de Saúde sobre pesquisa envolvendo seres humanos.

\section{ÁLISE ESTATÍSTICA}

Os dados foram apresentados através de esta- tística descritiva em média, desvio padrão, freqüência e percentual.

Foi utilizada a estatística descritiva para as variáveis, idade, estatura, massa corporal e IMC dos escolares. Para classificação do IMC e nível de atividade física e sua relação, entre os grupos, escolas públicas e privadas, foi utilizado o teste quiquadrado $\left(\mathrm{x}^{2}\right)$.

Foram utilizados, para os resultados, tabelas, gráficos e consideradas significativas diferenças com $p<0,05$. O software utilizado foi o SPSS, versão 15.0 .

\section{RESULTADOS}

Os resultados médios ( ), desvio-padrão (SD) (tabela 1), das variáveis antropométricas mostram a caracterização dos escolares. A idade dos adolescentes foi de 12,09 $\pm 0,80$; estatura, 1,53 $\pm 0,87$; massa corporal, 43,22 $\pm 10,09$ e IMC, 18,17 $\pm 2,85$.

TABELA 1. Valores médios ( ), desvio-padrão (SD) das variáveis antropométricas dos escolares.

\begin{tabular}{c|c}
\hline \multirow{2}{*}{ VARIÁVEIS } & TOTAL $(\mathbf{n}=797)$ \\
\cline { 2 - 2 } & $\bar{x} \pm$ SD \\
\hline IDADE & $12,09 \pm 0,80$ \\
\hline ESTATURA & $1,53 \pm 0,87$ \\
\hline MASSA CORPORAL & $43,22 \pm 10,09$ \\
\hline IMC & $18,17 \pm 2,85$ \\
\hline
\end{tabular}

Os dados antropométricos e nível de atividade física estão apresentados na tabela 2 em dois grupos: pública e privada. Foi feita uma análise descritiva para verificar se há diferenças significativas do IMC dos escolares das redes pública e privada, e uma correlação entre o IMC e o nível de atividade física. Os resultados da classificação do IMC (normal, sobrepesado e obeso) dos escolares mostram que há uma presença no excesso de peso, onde $12,3 \%$ são sobrepesados e $3,1 \%$ obesos, mostrando correlação com uma tendência mundial. Não houve diferença significativa entre as redes pública e privada, havendo homogeneidade entre os grupos.

Outro aspecto importante a ser estudado foi o nível de atividade física dos adolescentes de escolas públicas e privadas. Pelo teste qui-quadrado $\left(x^{2}\right)$, pode-se constatar que, na variável ativo, os alunos das escolas públicas, (19,4\%), são mais ati- 
vos que os das escolas privadas, $(5,5 \%)$. Outro resultado relevante mostra que a maioria dos alunos do ensino público, $19,4 \%$, são ativos e os alunos do ensino privado, $8,5 \%$, revelam-se sedentários, verificando-se aí um resultado altamente inverso. Analisando-se os estudantes de todas as escolas, foi notado que $25,2 \%$ são insuficientemente ativos, denotando uma tendência ao sedentarismo.

TABELA 2. Classificação do IMC e nível de atividade física dos adolescentes em escolas públicas e privadas.

\begin{tabular}{|c|c|c|c|c|c|c|}
\hline \multirow[t]{2}{*}{$\begin{array}{l}\text { Classificação do } \\
\text { IMC }\end{array}$} & \multicolumn{2}{|c|}{$\begin{array}{l}\text { Pública } \\
(\mathrm{n}=580)\end{array}$} & \multicolumn{2}{|c|}{$\begin{array}{l}\text { Privada } \\
(\mathrm{n}=217)\end{array}$} & \multicolumn{2}{|c|}{$\begin{array}{c}\text { Total } \\
(\mathrm{n}=797)\end{array}$} \\
\hline & $F$ & $\%$ & f & $\%$ & f & $\%$ \\
\hline Normal & 492 & 61,7 & 182 & 22,8 & 674 & 84,6 \\
\hline Sobrepeso & 68 & 8,5 & 30 & 3,8 & 98 & 12,3 \\
\hline Obeso & 20 & 2,5 & 5 & 0,6 & 25 & 3,1 \\
\hline Total & 580 & 72,8 & 217 & 27,2 & 797 & 100,0 \\
\hline \multirow[t]{2}{*}{$\begin{array}{c}\text { Nível de } \\
\text { Atividade Física }\end{array}$} & \multicolumn{2}{|c|}{$\begin{array}{l}\text { Pública } \\
\text { ( } \mathrm{n}=580)\end{array}$} & \multicolumn{2}{|c|}{$\begin{array}{l}\text { Privada } \\
(\mathrm{n}=217)\end{array}$} & \multicolumn{2}{|c|}{$\begin{array}{c}\text { Total } \\
(n=797)\end{array}$} \\
\hline & $F$ & $\%$ & f & $\%$ & $f$ & $\%$ \\
\hline Inativo & 130 & $16,3 \%$ & 68 & $8,5 \%$ & 198 & $24,8 \%$ \\
\hline $\begin{array}{l}\text { Insuficientemente } \\
\text { Ativo }\end{array}$ & 144 & $18,1 \%$ & 57 & $7,2 \%$ & 201 & $25,2 \%$ \\
\hline $\begin{array}{l}\text { Moderadamente } \\
\text { Ativo }\end{array}$ & 151 & $18,9 \%$ & 48 & $6,0 \%$ & 199 & $25,0 \%$ \\
\hline Ativo & 155 & $19,4 \%$ * & 44 & $5,5 \%$ & 199 & $25,0 \%$ \\
\hline Total & 580 & $72,8 \%$ & 217 & $27,2 \%$ & 797 & $100 \%$ \\
\hline
\end{tabular}

* Efeito significativo na variável ativo nos grupo Pública e Particular $p>0,03$.

Pode-se observar, na figura 1 , a relação entre o nível de atividade física (NAF) e a classificação do IMC em adolescentes de escolas públicas. Os resultados mostram que há mais alunos classificados como normais, com o nível de atividade física ativo, (23,6\%). Em insuficientemente ativos, os alunos com sobrepeso totalizam $(3,4 \%)$ e os obesos $(1,0 \%)$; verificando-se, desta forma, que os adolescentes com excesso de peso têm predisposição a serem sedentários.

FIGURA 1. NAF e classificação do IMC em adolescentes de escolas públicas.

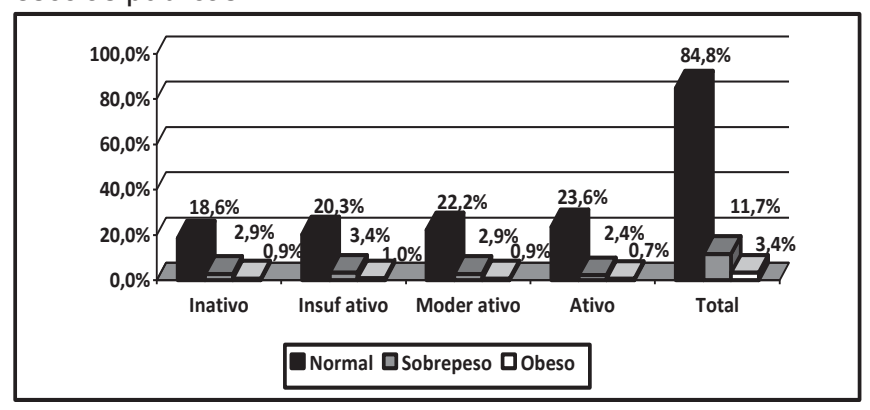

A figura 2 mostra a relação entre o nível de atividade física (NAF) e a classificação do IMC em adolescentes de escolas privadas. Os resultados revelam que há mais adolescentes classificados como normais, com o nível de atividade física inativo, (25,8\%), resultado oposto ao das escolas públicas, analisadas na figura 1. Ao observar os estudantes com sobrepeso, verificou-se que a maioria possui o nível de atividade física classificada como inativo, (4,6\%), enquanto que os obesos, tanto ativo quanto moderadamente ativo, atingem o percentual de $(0,9 \%)$, indicando que, independente da classificação do IMC, os adolescentes das escolas privadas mostramse sedentários.

FIGURA 2. NAF e classificação do IMC em adolescentes de escolas privadas.

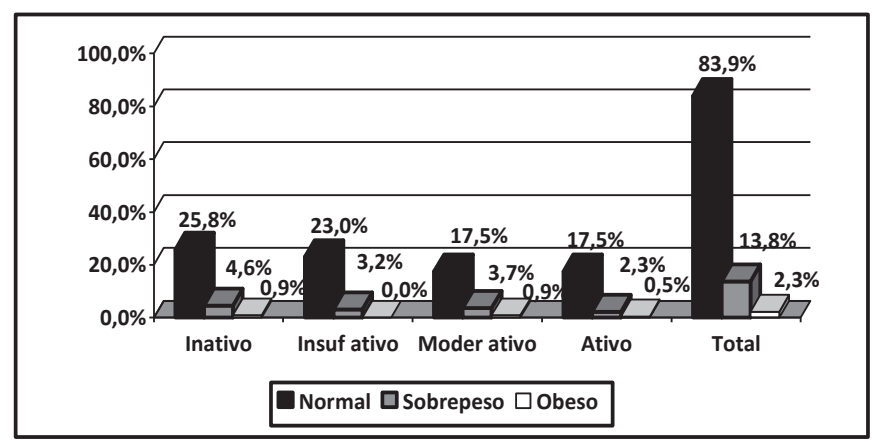

A figura 3, por fim, mostra a relação entre o nível de atividade física (NAF) e a classificação do IMC em adolescentes do total das escolas. Os resultados revelam que os adolescentes com a classificação normal são, em sua maioria, ativos, $(22,0 \%)$; os sobrepesados inativos $(3,4 \%)$ e os insuficientemente ativos (3,4\%), enquanto os obesos inativos $(0,9 \%)$ e os moderadamente ativos $(0,9 \%)$, verificando-se, assim, que os adolescentes com excesso de peso, independentemente de estudarem em escolas públicas ou privadas, são sedentários.

FIGURA 3. NAF e classificação do IMC em adolescentes do total de escolas..

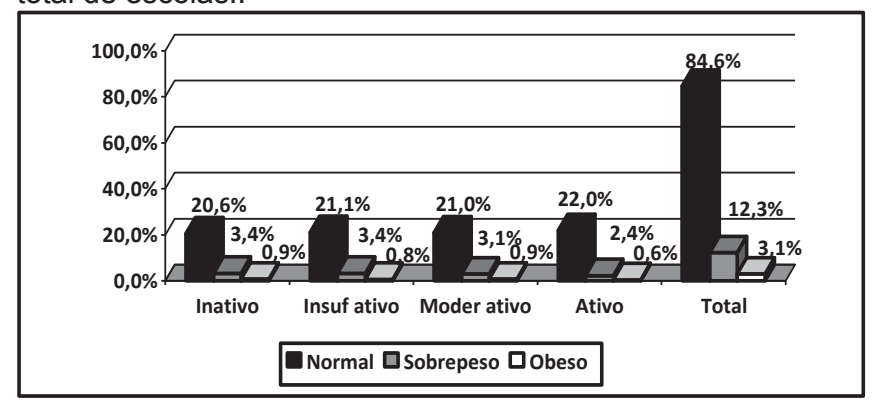




\section{DISCUSSÃO}

O presente estudo mostra uma prevalência de sobrepeso e obesidade, com $12,3 \%$ de sobrepesados e $3,1 \%$ de obesos, de acordo com a encontrada na literatura, independente do critério de diagnóstico utilizado, confirmando a magnitude e gravidade que o problema vem assumindo entre os escolares de todo o Brasil. O estudo com o objetivo de avaliar o estado nutricional e o consumo alimentar de adolescentes dos sexos masculino e feminino, matriculados em um Centro de Juventude da cidade de São Paulo, mostra a prevalência do excesso de peso, com $7,8 \%$ de sobrepeso e $11,8 \%$ com risco de sobrepeso ${ }^{(11)}$. Em outro estudo, com o propósito de avaliar o estado nutricional e a maturação sexual de adolescentes de 11 a 15,9 anos, de níveis socioeconômicos diferentes, acharam um valor de $6,6 \%$ com sobrepeso e $9,2 \%$ obesos ${ }^{(19)}$. O estudo com o objetivo de conhecer os hábitos alimentares e o estado nutricional dos adolescentes de escola privada do município de Viçosa-MG mostrou um índice de $10,3 \%$ de excesso de peso nos escolares (17), corroborando com nossos achados.

Os achados referentes ao tipo de escola, pública $(8,5 \%$ e $2,5 \%)$ e privada $(3,8 \%$ e $0,6 \%)$, não mostraram diferenças significativas na prevalência de sobrepeso ou obesidade. Resultado divergente ao estudo que teve como propósito de determinar a prevalência de sobrepeso e obesidade em adolescentes escolares do Município de Fortaleza, Brasil, e estimar a diferença entre a prevalência nas escolas públicas e privadas segundo sexo e faixa etária (adolescência precoce e tardia), onde se observou maior freqüência de sobrepeso/obesidade nas escolas privadas (587) quando comparadas às públicas (571) e a prevalência de sobrepeso/obesidade para toda a amostra foi de $19,5 \%{ }^{(7)}$, valor maior que o dos nossos resultados com sobrepeso e obesidade de $12,3 \%$ e $3,1 \%$.

Outro estudo também mostrou diferenças significativas entre escolas ao avaliar a associação entre obesidade e dislipidemias em adolescentes do ensino público e privado de Campina Grande-PB, Brasil, onde o percentual de sobrepeso, na amostra, foi de $14,4 \%$, resultado próximo ao do nosso estudo, mas a categoria deste estado nutricional foi maior na escola pública $(15,8 \%)$ do que na privada $(12,9 \%)^{(8)}$.

O nível de atividade física dos adolescentes analisados, considerando-se toda a amostra, 25,2\% $(n=201)$ dos adolescentes, foi classificado como insuficientemente ativos, sendo que, nas escolas privadas, os alunos, em sua maioria, eram inativos $8,5 \%$ e, nas escolas públicas, ativos 19,4\%. Esse resultado foi semelhante à pesquisa realizada na região serrana, em Santa Catarina, onde foi verificado o nível de atividade física de adolescentes de escolas públicas e privadas. Pela análise do nível habitual de atividade física dos adolescentes, por rede de ensino, foram detectadas diferenças estatisticamente significativas $(p<0,05)$, demonstrando uma maior proporção de adolescentes da rede pública (estadual $64,3 \%$ e municipal 56,5\%) no nível ativo quando comparados aos da rede particular, $46,9 \%$. Os adolescentes da rede particular de ensino também foram classificados como muito inativos, $28,1 \%$, quando comparados aos da rede pública (estadual 15,0\% e municipal 16,0\%) $(p<0,05)$ (5). Outra pesquisa encontrou resultados parecidos com os dos nossos estudos, a que teve como propósito relacionar o nível de atividade física à prevalência de obesidade e ao perfil lipídico de adolescentes escolares do município de Rio Verde-GO. $O$ resultado mostra que os adolescentes da rede pública de ensino são mais ativos, $81,78 \%$, quando comparados com os da rede privada, $71,03 \%$ $(p=0,0000)^{(27)}$. Divergente do estudo onde os alunos de classes econômicas mais elevadas (25\%) tiveram níveis de atividade física maior que os de classe econômica menos elevada $(10,6 \%)^{(18)}$.

Nossos resultados mostram que, independente da escola em que estudam, os adolescentes com sobrepeso e obesidade são, em sua maioria, sedentários. Nas escolas públicas, os sobrepesados $(3,4 \%)$ e obesos $(1,0 \%)$ são insuficientemente ativos; nas escolas privadas, os sobrepesados $(4,6 \%)$ e obesos $(0,9 \%)$ são inativos e, no total de escolas, os sobrepesados são inativos $(3,4 \%)$ e insuficientemente ativos $(3,4 \%)$ e os obesos, inativos $(0,9 \%)$ e moderadamente ativos $(0,9 \%)$. Em relação ao total de escolas apenas os estudantes classificados como normais das escolas privadas são sedentários. No estudo com o propósito de des- 
crever a prevalência de obesidade em alunos de escolas públicas e particulares, comparando com a influência das variáveis sexo, idade, nível socioeconômico, atividade física e consumo de alimentos; verificaram que os alunos com obesidade eram em sua maioria sedentários, escolas públicas $(57,3 \%)$ e particulares $(55,3 \%)^{(14)}$, resultados semelhantes aos da nossa pesquisa. A pesquisa com o propósito de analisar a relação entre obesidade em escolares e atividade física e horas de sono de estudantes, observaram que cerca de $75 \%$ da rotina diária das crianças estão distribuídas entre horas de sono e permanência sentado ${ }^{(12)}$. Foram também constatadas que o grupo sobrepesado tem um tempo de permanência sentado maior que o grupo normal $(p<0,05)$ e que há uma tendência da prática desportiva ser mais freqüente nas crianças normais do que nas com sobrepeso.

No estudo, objetivando avaliar o nível de atividade física, qualidade alimentar do lanche escolar e composição corporal de estudantes do ensino fundamental e médio, observaram que os estudantes classificados como normais $(n=156)$ e sobrepesados ( $n=83$ ), são em sua maioria insuficientemente ativos, não apresentando diferenças significativas $(p<0,05)$ entre os grupos ${ }^{(24)}$. Da mesma forma, o estudo que teve como objetivo de determinar a prevalência de obesidade entre escolares, bem como investigar sua associação com possíveis fatores que possam favorecer o excesso de peso corporal, confirmaram a associação entre o tempo de conduta sedentária e a prevalência de sobrepeso ou obesidade $(31,4 \%)$, mostrando que adolescentes que permanecem em média mais de 4 horas e 30 minutos por dia em conduta sedentária têm maiores chances de apresentar sobrepeso ou obesidade, corroborando com nossos resultados ${ }^{(29)}$.

O sedentarismo é uma das principais causas da obesidade ${ }^{(15)}$. A inatividade causa 1,9 milhão de mortes por ano no mundo e, infelizmente, tem aumentado substancialmente esta inatividade entre os jovens ${ }^{(21,23)}$.

Para a prevenção do sobrepeso e obesidade é imprescindível o aumento da atividade física, menos tempo de televisão e uma alimentação saudável ${ }^{23,25)}$. A inatividade física causa o excesso de peso; portanto, quanto mais cedo existir a mudança do estilo de vida sedentário para o ativo, mais fácil será mudar os hábitos dos jovens, contribuindo para uma vida mais saudável futuramente.

\section{CONCLUSÃO}

A vida moderna tem favorecido o desenvolvimento de obesidade em jovens na medida em que, pela violência urbana e falta de espaço, os jovens são desestimulados a brincar e praticar esportes nas ruas, praças e espaços públicos; incentivados a desenvolver atividades com jogos eletrônicos e computador, a assistir televisão. Esses fatos vêm contribuindo para o aumento do sedentarismo e, conseqüentemente, elevando os níveis de sobrepeso e obesidade.

Pelo estudo desenvolvido em Taguatinga/DF, conclui-se que os escolares das redes de ensino pública e privada apresentam-se em grande parte sedentários, sendo que os alunos das escolas particulares destacam-se quanto ao baixo nível de atividade física, caracterizando-se como menos ativos que os alunos das escolas públicas. O excesso de peso encontrado nos adolescentes das redes de ensino foi elevado. E, em relação à associação entre a classificação do IMC e nível de atividade física, os adolescentes com excesso de peso, independentemente da escola em que estudam, são sedentários. O mesmo acontece com os adolescentes classificados como normais das escolas privadas, que foram considerados sedentários.

\section{REFERÊNCIAS BIBLIOGRÁFICAS}

1. Associação Brasileira para o Estudo da Obesidade e Síndrome Metabólica (ABESO). Obesidade em notícia. 2008. Disponível em: http://www.abeso.org.br/reportagens/dia_nacional_sedentarismo.htm Acesso em 02/06/2008.

2. Ainsworth BE, Haskell WL, Whitt MC, Irwin ML, Swartz AM, Strath SJ, O'brien WL, et al. Compendium of physical activities: an update of activity codes and met intesities. Medicine and Science in sports and exercise. 2000.

3. American College of Sports Medicine. Calling on physicians to assess and review every patient's physical activity program at every visit. Public. 2008. Disponível em: http://www.exerciseismedicine.org/public.htm Acessado em: 29/06/2008. 
4. Araújo TL, Lopes MVO, Cavalcante TF, Guedes NG, Moreira RP, Chaves ES, Silva VM. Análise de indicadores de risco para hipertensão arterial em crianças e adolescentes. Revista de Escola de Enfermagem da USP. Vol 42(1):120-6. 2008.

5. Arruda ELM, Lopes AS. Gordura corporal, nível de atividade física e hábitos alimentares de adolescentes da região serrana de Santa Catarina, brasil. Revista Brasileira de Cineantropometria e Desempenho Humano. Vol 9(1):05-11, 2007.

6. Barros MVG, Nahas MV. Medidas da atividade física: Teoria e aplicação em diversos grupos populacionais. Londrina: Midiograf, 2003. 160 p.

7. Campos LA, Leite AJM, Almeida PC. Prevalência de sobrepeso e obesidade em adolescentes escolares do município de Fortaleza, Brasil. Revista Brasileira de Saúde Materno Infantil. Recife, vol 7 (2): 183-190, abr. / jun., 2007.

8. Carvalho DF, Paiva AA, Melo ASO, Ramos AT, Medeiros JS, Medeiros CCM, Cardoso MAA. Perfil lipídico e estado nutricional de adolescentes. Revista Brasileira de Epidemiologia. Vol 10(4): 491-8, 2007.

9. Centers for Disease Control and Prevention (CDC)/ National Center for Chronic Disease Prevention and Health Promotion. Physical Activity for Everyone Physical Activity and Health. DNPAO CDC. . 2008. http://www. cdc.gov/nccdphp/dnpa/physical/everyone/health/ Visitado em 02/06/2008.

10. Cole TJ, Bellizzi MC, Flegal KM, Dietz WH. Establishing a standard definition for child overweight and obesity worldwide: international survey. British Medical Journal. V.320, May 2000. Disponível em: http://www. pubmedcentral. . ih. gov/articlerender.fcgi? artid=27365 Acesso em: 07 fev. 2008.

11. Garcia GCB, Gambardella AMD; Frutuoso MFP. Estado nutricional e consumo alimentar de adolescentes de um centro de juventude da cidade de São Paulo. Revista de Nutrição. Campinas, 16(1):41-50, jan./mar., 2003.

12. Giugliano R, Carneiro EC. Fatores associados $\ddagger$ obesidade em escolares. Jornal de Pediatria (Rio J); 80(1):17-22, 2004.

13. Gonçalves H, Hallal PC, Amorim TC, Araújo CLP, Menezes AMB. Fatores socioculturais e nível de atividade física no início da adolescência. Revista Panamericana de Salud Pública. Vol 22(4), 2007.

14. Leão LSCS, Araújo, LMB, Moraes LTLP, Assis AM. Prevalência de Obesidade em Escolares de Salvador, Bahia. Arquivo Brasileiro de Endocrinologia e Metabologia. vol $47 n^{\circ} 2$ Abril 2003.
15. Ministério da Saúde. Guia alimentar para a população brasileira: promovendo a alimentação saudável / Ministério da saúde, Secretaria de atenção à saúde, coordenação-geral da política de alimentação e nutrição - Brasília, 2006.

16. Ministério da Saúde. Indicadores de morbidade e fatores de risco - D.24 Taxa de prevalência de excesso de peso. 2008. Disponível em: http://tabnet.datasus.gov. br/cgi/idb2006/d24b.htm Acesso em 02/06/2008.

17. Novaes JF; Priore SE, Franceschini SCC. Estado nutricional e hábitos alimentares de adolescentes de escola privada. Bioscience Journal. Uberlândia. V.20, n.1, p. $97-$ 105, Jan/Apr. 2004.

18. Nunes MMA, Figueiroa JN, Alves JGB. Excesso de peso, atividade física e hábitos alimentares entre Adolescentes de diferentes classes econômicas em Campina Grande (PB). Revista de Associação Medica Brasileira. Vol 53(2): 130-4. 2007.

19. Oliveira CS, Veiga GV. Estado nutricional e maturação sexual de adolescentes de uma escola pública e de uma escola privada do Município do Rio de Janeiro. Revista de Nutrição. Campinas, 18(2):183-191, mar./abr., 2005.

20. Organização Mundial de Saúde (OMS). Notícias: OMS defende abordagem similar contra o cigarro no combate à obesidade. 2008. Disponível em: http:// dtr2004.saude.gov.br/nutricao/noticia/noticia_ret_detaIhe.php?cod=558 Acesso em 02/06/2008.

21. Organização Pan-Americana de Saúde (OPAS). Doenças crônico-degenerativas e obesidade: estratégia mundial sobre alimentação saudável, atividade física e saúde. / Organização Pan-Americana de Saúde - Brasília. 2003.

22. Organização Pan-Americana de Saúde (OPAS). Informativos: Lançamento do Vigitel 2007 causa grande impacto na mídia. 2008. Disponível em: http://www. opas.org.br/mostrant.cfm?codigodest $=372$ Acesso em 02/06/2008.

23. Ornelas IJ, Perreira KM, Ayala GX. Parental Influences on adolescent physical activity: a longitudinal Study. International Journal of Behavioral Nutrition and Physical Activity. 4:3. 2007.

24. Pierine DT, Carrascosa A.PM, Fornazari AC, Watanabe MT, Catalani MCT, Fukuju MM, Silva GN, Maestá N. Composição corporal, atividade física e consumo alimentar de alunos do ensino fundamental e médio. Motriz, Rio Claro, v.12 n.2 p.113-124, mai./ago. 2006. 
25. Perrim EM, Finkle JP, Benjamin JT. Obesity prevention and the primary care pediatrician's office. Current Opinion in Pediatrics. jun v.19(3), p.354-61, 2007.

26. Petroski EL. Antropometria Técnicas e padronizações. 3. ed. rev. e ampl. Blumenau, SC: Nova Letra, 2007.

27. Silva RCD. Obesidade, perfil lipídico e sua relação com o nível de atividade física de adolescentes escolares. Dissertação de mestrado em Ciências da Saúde da UnB. Brasília 2007.

28. Soares NT. Um novo referencial antropométrico de crescimento: significados e implicações. Revista de Nutrição. Campinas. v. 16, n.1, jan./ mar. 2003.

29. Suñé FR, Dias-da-costa JS, Olinto MTA, Pattussi MP. Prevalência e fatores associados para sobrepeso e obesidade em escolares de uma cidade no Sul do Brasil. Caderno de Saúde Pública, Rio de Janeiro, 23(6):13611371, jun, 2007.
30. Us Department of Health and Human Services. Physical Activity and Health: A Report of the Surgeon General. Atlanta, GA: US Department of Health and Human Services. Centers for Disease Control and Prevention, National Center for Chronic Disease Prevention and Health Promotion, 1996.

31. Word Health Organization (WHO). Obesity and overweight. 2008. Disponível em: http://www.who.int/ mediacentre/factsheets/fs311/en/index.html Acesso em 02/06/2008.

\section{ENDEREÇO PARA CORRESPONDÊNCIA:}

Rua Jonas Vanucci Lins $n^{\circ} 50$ - Bloco 1 - Ap 404 - Jardim Paquetá - Belo Horizonte -MG. CEP: 31330-640 Fone: (31) 8364-4114 E-mail: lubaiucha@hotmail.com 